\title{
Utilità dell'autopsia in Medicina Interna: discrepanze tra diagnosi clinica e diagnosi autoptica
}

\author{
Value of autopsy in Internal Medicine: discrepancies between clinical \\ and postmortem diagnosis
}

\section{Fabrizio Tiratterra*, Valeria Pignatelli, Alberto Placido, Agostino Valenti, Umberto Recine}

UOC Medicina Interna, Ospedale Santo Spirito, Roma

Ricevuto il 13 settembre 2011; accettato il 27 ottobre 2011

Disponibile online il 13 marzo 2012

\section{KEYWORDS \\ Clinical diagnosis; \\ Autopsy; \\ Diagnostic errors.}

\begin{abstract}
Summary
Introduction: The number of autoptic controls is progressively dropping worldwide during the last decades. Several social, cultural and normative causes may underlie this phenomenon. However, a hidden cause may be represented by the scepticism of physicians about the gain in information given by this practice. There is in fact a general thinking that available diagnostic techniques may be adequate for a correct diagnosis, even in the case of a mortal outcome. Nevertheless, several studies have shown quite relevant discrepancies between premortem and postmortem diagnosis. In this study, the authors evaluate the accuracy of clinical diagnosis in diseases leading the patient to death.

Materials and methods: Retrospective analysis including all patients died in the Department of Internal Medicine of a General Hospital in Rome during a three year time period (1st January 2007-31 December 2009). Age, sex, period of hospitalization, clinical diagnosis, autoptic diagnosis, cause of death, and level of discrepancy between clinical diagnosis and autoptic control have been collected. Main diagnoses have been classified as follows: (1) discrepancy with a possible influence on survival; (2) discrepancy with no or questionable influence on survival. Results: Ninety-two cases (42 males, 50 females; mean age 79.3 years) have been included. Thirty-four main diagnoses (36.9\%) have been classified as discrepant (15.2\% classified as type 1 discrepancy and 21.7 as type 2 ).

Discussion: A substantial discrepancy between clinical diagnosis and autoptic control is confirmed by the current study, which supports the role of autopsy as a tool for the improvement of medical practice.
\end{abstract}

(c) 2012 Elsevier Srl. All rights reserved.

\footnotetext{
* Corrispondenza: via del Pellegrino 177 - 00186 Roma.

E-mail: f.tiratterra@virgilio.it (F. Tiratterra).
} 


\section{Introduzione}

La rilevanza della autopsia medica (riscontro diagnostico) nella pratica clinica è oggetto di dibattito nella comunità scientifica da oltre un decennio. L'autopsia è ritenuta l'esame clinico più accurato per definire la causa di morte e consente di valutare la precisione della diagnosi clinica; esiste un ampio consenso sulla sua utilità nel percorso formativo degli studenti in Medicina e dei medici. Tuttavia, la pratica dell'autopsia medica è in progressiva diminuzione nel corso degli anni in tutto il mondo: il tasso autoptico, cioè il rapporto tra il numero di autopsie effettuate e il numero totale dei decessi, negli ospedali europei e americani negli anni Sessanta era intorno al 60\%, mentre alla fine degli anni Novanta era pari al $10 \%$ o meno [1]. Per quanto riguarda la realtà italiana, il tasso autoptico di sei ospedali della Regione Piemonte negli anni 1995-'97 era compreso tra il 7 e il 13\% [2].

Le cause del declino della pratica dell'autopsia medica sono molteplici: tra queste vi sono motivi legislativi (quali l'obbligo di ottenere il consenso dei parenti previsto in alcuni Paesi, come la Francia), motivazioni socioculturali, il timore dei medici di essere coinvolti in dispute medico-legali, il costo della procedura. Tuttavia, un motivo ancora più rilevante sembra essere lo scetticismo dei medici circa il valore del riscontro autoptico. Infatti, molti clinici ritengono che le moderne tecniche di diagnostica per immagini e le altre tecnologie disponibili siano adeguate per diagnosticare con precisione una malattia e per capire la causa di morte. Sono invece numerosi gli studi che fin dal 1912 [3] hanno dimostrato significative discrepanze tra diagnosi clinica e diagnosi autoptica. Tale discrepanza sembra persistere nonostante l'evoluzione tecnologica. Alcuni di questi studi sono stati focalizzati su pazienti deceduti in reparti di Medicina Interna, tuttavia non sono a nostra conoscenza analoghi studi svolti in divisioni di Medicina Interna di ospedali italiani.

Scopo del presente lavoro è valutare l'accuratezza della diagnosi clinica in un reparto ospedaliero di Medicina Interna assumendo la diagnosi autoptica come standard di riferimento, e identificare i fattori che potrebbero influenzare negativamente l'accuratezza diagnostica.

\section{Materiali e metodi}

Lo studio, di tipo retrospettivo, è stato condotto nell'Unità Operativa Complessa (UOC) di Medicina Interna dell'Ospedale
Santo Spirito di Roma, un ospedale non universitario. Sono stati inclusi tutti i casi di pazienti deceduti nel reparto di Medicina Interna sottoposti ad autopsia nel periodo compreso tra il $1^{\circ}$ gennaio 2007 e il 31 dicembre 2009.

Per ogni caso sono stati registrati i seguenti dati: età, sesso, durata della degenza, diagnosi clinica, diagnosi autoptica, causa di morte, grado di discrepanza. La diagnosi clinica è stata definita sulla base della diagnosi preautoptica formulata dai clinici al momento della richiesta dell'autopsia oppure, in assenza di questa, sulla base della diagnosi che era stata indicata nella cartella clinica. La diagnosi autoptica è stata acquisita dal referto dell'esame autoptico. Tutte le autopsie includevano analisi istopatologiche.

Il grado di discrepanza è stato catalogato in 5 classi (tab. 1), in accordo con l'approccio di Goldman et al. [4] modificato da Battle et al. [5]. In caso di presenza di più di una discrepanza nello stesso caso, questo veniva classificato attribuendogli la maggiore discrepanza tra quelle rilevate.

Il coordinatore della ricerca ha identificato tutti i casi nei quali era presente una discrepanza tra diagnosi clinica e autoptica; successivamente tutti i casi discrepanti sono stati analizzati da ciascuno dei ricercatori che ha attribuito in modo individuale e indipendente il grado di discrepanza. Nei casi di divergenza il grado di discrepanza è stato attribuito in un consensus tra i ricercatori.

Le diagnosi sono state classificate in accordo con l'International Classification of Disease $9^{\text {th }}$ Edition Clinical Modification (ICD 9 CM).

\section{Risultati}

Nel periodo oggetto dello studio, nella UOC di Medicina Interna sono deceduti 289 soggetti e sono state eseguite 92 autopsie (tasso autoptico: $31,8 \%$ ).

Sono stati quindi studiati 92 casi ( 50 femmine, 42 maschi). L'età media è risultata pari a 79,3 anni (range: 47-100 anni). In accordo con la classificazione esemplificata in tabella 1:

- le diagnosi principali discrepanti sono risultate $34(36,9 \%)$, $14(15,2 \%)$ di Classe 1 e 20 (21,7\%) di Classe 2;

- le diagnosi secondarie discrepanti sono state 23 (25,1\%): 18 $(19,6 \%)$ di Classe 3 e $5(5,4 \%)$ di Classe 4 ;

- non è stata riscontrata alcuna discrepanza tra diagnosi clinica e diagnosi autoptica in 35 casi $(38,1 \%)$ (tab. 2$)$.

Tabella 1 Classificazione delle discrepanze.

\begin{tabular}{lll}
\hline Classe & Definizione & Esempi \\
\hline 1 & $\begin{array}{l}\text { Diagnosi principale discrepante, l'identificazione } \\
\text { della quale avrebbe potuto influenzare la sopravvivenza }\end{array}$ & $\begin{array}{l}\text { Broncopolmonite in soggetto deceduto per infarto } \\
\text { intestinale }\end{array}$ \\
\hline 2 & $\begin{array}{l}\text { Diagnosi principale discrepante, l'identificazione della } \\
\text { quale avrebbe potuto avere dubbia o nessuna influenza } \\
\text { sulla sopravvivenza }\end{array}$ & $\begin{array}{l}\text { Infarto polmonare in soggetto con insufficienza } \\
\text { multiorgano e sindrome da allettamento }\end{array}$ \\
\hline 3 & $\begin{array}{l}\text { Diagnosi secondaria discrepante che avrebbe potuto } \\
\text { essere formulata prima della morte }\end{array}$ & $\begin{array}{l}\text { Adenocarcinoma del coledoco in soggetto deceduto } \\
\text { per insufficienza cardiaca }\end{array}$ \\
\hline 4 & Diagnosi secondaria discrepante che non avrebbe & Diverticolosi del sigma in soggetto deceduto per \\
& potuto essere formulata prima della morte & broncopolmonite bilaterale \\
\hline 5 & Completo accordo tra diagnosi clinica e autoptica & \\
\hline
\end{tabular}


Tabella 2 Concordanza tra diagnosi clinica e diagnosi autoptica.

\begin{tabular}{lll}
\hline & Numero di casi & \% su totale casi \\
\hline Classe 1 & 14 & 15,2 \\
\hline Classe 2 & 20 & 21,7 \\
\hline Classe 3 & 18 & 19,6 \\
\hline Classe 4 & 5 & 5,4 \\
\hline Classe 5 & 35 & 38,1 \\
\hline Totale & 92 & 100 \\
\hline
\end{tabular}

Tabella 3 Diagnosi di morte.

\begin{tabular}{ll}
\hline & Numero di casi \\
\hline Broncopolmonite & 33 \\
\hline Scompenso cardiaco & 19 \\
\hline Neoplasie (solide ed ematologiche) & 13 \\
\hline Embolia polmonare & 6 \\
\hline Sepsi & 6 \\
\hline Infarto miocardico acuto & 4 \\
\hline Ictus cerebrale & 4 \\
\hline Shock postemorragico & 2 \\
\hline Pielonefrite acuta & 1 \\
\hline Rottura di aneurisma dell'aorta toracica & 1 \\
\hline Cardiopatia ischemica & 1 \\
\hline Infarto intestinale & 1 \\
\hline Amiloidosi cardiaca & 1 \\
\hline Totale casi & 92 \\
\hline
\end{tabular}

Genere e durata del ricovero non sono risultati correlati con l'incidenza di diagnosi principali discrepanti.

Le diagnosi autoptiche che hanno originato gli errori di tipo 1 sono risultate le seguenti:

- insufficienza cardiaca (3 casi);

- embolia polmonare (2 casi);

- polmonite (2 casi);

- sepsi (2 casi);

- infarto miocardico acuto (1 caso);

- rottura di aneurisma dell'aorta toracica (1 caso);

- infarto intestinale (1 caso);

- shock postemorragico (1 caso);

- linfoma non Hodgkin (1 caso).

Le diagnosi di morte riscontrate nei 92 casi sono riportate nella tabella 3 .

\section{Discussione}

Nonostante il progresso delle conoscenze mediche e il tumultuoso sviluppo delle tecnologie diagnostiche, numerosi studi hanno dimostrato la persistenza di un consistente numero di errori diagnostici svelati dal riscontro autoptico. Nel nostro studio la discrepanza diagnostica relativa alla diagnosi principale è stata pari al 36,9\%.

La percentuale di discrepanza di grado 1 e 2 da noi riscontrata risulta più elevata rispetto ad altri studi. In una recente review Shojania et al. [6] hanno rilevato un rapporto mediano di errore pari al 23,5\% (range 4,1$49,8 \%$ ) per gli errori maggiori (definiti come discrepanza di grado 1 e 2).

Il rapporto di discrepanza da noi rilevato risulta invece sostanzialmente sovrapponibile a quello di altri studi focalizzati, come il nostro, esclusivamente su pazienti medici: tali studi hanno rilevato una percentuale di diagnosi maggiori discrepanti (grado 1 e 2) compresa tra il 34\% e il 41\% [7-10]

Alcune possibili cause rendono ragione del valore relativamente alto di discrepanza che abbiamo riscontrato. In primo luogo l'età media molto elevata, notevolmente maggiore di quella di altri studi analoghi riguardanti pazienti sia medici sia chirurgici. Numerosi studi hanno infatti documentato un aumento degli errori diagnostici nella popolazione più anziana [5,11]. L'origine di tale aumento nei pazienti anziani può essere dovuta all'intersecarsi di più cause. Una di esse, a nostro giudizio, è la presentazione spesso atipica di sintomi e segni negli anziani, nei quali prevalgono sintomi sistemici e generici come l'astenia, la sensazione di malessere generale, l'anemia, come pure la difficoltà di ottenere informazioni utili circa caratteristiche e sede di un'eventuale sintomatologia dolorosa, anche per la non infrequente presenza di disturbi cognitivi.

Un altro motivo riteniamo sia la consapevole decisione dei clinici di non eseguire ulteriori accertamenti diagnostici di tipo invasivo e con possibili complicazioni in pazienti in condizioni generali scadute e con malattie sistemiche avanzate, nei quali l'eventuale migliore definizione diagnostica del quadro clinico non modificherebbe le possibili strategie terapeutiche. Un altro elemento che può aver contribuito a determinare il grado di discrepanza riscontrato è l'elevata frequenza di polipatologia nei casi studiati. che può aver reso più difficoltosa l'identificazione della causa di morte in alcuni di essi.

Le diagnosi discrepanti di Classe 1, quelle la cui identificazione avrebbe potuto influenzare la sopravvivenza, sono state 14 (15,2\%). La più frequente diagnosi mancata è stata quella di insufficienza cardiaca, in 3 casi. L'embolia polmonare è stata la causa di discrepanza di Classe 1 in 2 casi, mentre l'infarto miocardico acuto non è stato diagnosticato in 1 caso. Tali diagnosi risultano comuni cause di discrepanza anche in altri studi [1]. Queste condizioni cliniche presentano, infatti, uno spettro di sintomi comuni e soggetti anziani con polipatologia frequentemente presentano un elevato rischio di sviluppare ognuna di queste patologie. La polmonite non è stata diagnosticata in 2 casi. Ciò è in accordo con numerosi altri studi [9,12-15] che hanno rilevato come la polmonite sia una frequente causa di diagnosi mancata. La polmonite nosocomiale, infatti, è spesso difficile da riconoscere nei soggetti anziani in condizioni cliniche compromesse. In entrambi i casi di polmonite, febbre e leucocitosi erano assenti. La sepsi è stata responsabile di 2 casi di mancata diagnosi. Altri autori hanno enfatizzato il ruolo delle setticemie come causa di morte non diagnosticata [16]: ciò è stato posto in relazione con l'aumentata esposizione dei pazienti a terapie antibiotiche ad ampio spettro. Le neoplasie maligne sono risultate causa di discrepanza di Classe 1 in 
un caso (linfoma non Hodgkin). Nei restanti 3 casi le diagnosi mancate sono state infarto intestinale, rottura di aneurisma dell'aorta toracica, shock ipovolemico postemorragico.

Vi sono alcuni limiti nel presente studio. In primo luogo è uno studio retrospettivo e ciò potrebbe aver ridotto la precisione nella raccolta dei dati. Inoltre potrebbe esserci stato un bias nella selezione dei casi sottoposti a esame autoptico: infatti, l'esecuzione del riscontro autoptico potrebbe essere stata richiesta nei casi nei quali vi era una maggiore incertezza diagnostica e ciò potrebbe aver influenzato l'incidenza delle discrepanze. Alcuni studi $[16,17]$ hanno però evidenziato la scarsa abilità dei clinici nell'identificare i casi che possono riservare diagnosi inattese.

Un ulteriore limite è intrinsecamente legato all'utilizzazione della classificazione di Goldman et al. [4], la più usata in letteratura, poiché richiede un giudizio relativo all'impatto della discrepanza tra diagnosi clinica e autoptica sulla sopravvivenza del paziente che può essere difficile determinare.

\section{Conclusioni}

Il nostro studio conferma che, nonostante il continuo perfezionamento delle tecnologie diagnostiche, formulare la diagnosi corretta rimane un esercizio difficile e commettere errori risulta inevitabile. La procedura dell'autopsia diagnostica continua a contribuire al miglioramento della pratica clinica consentendo di verificare l'accuratezza della propria attività professionale. Nei casi con diagnosi dubbia, nonostante l'utilizzazione delle tecnologie diagnostiche più evolute, è opportuno ricorrere al riscontro diagnostico. Infatti, il riconoscere e il comprendere gli errori commessi risulta fondamentale per il miglioramento personale e per quello della qualità delle cure.

\section{Conflitto di interessi}

Gli autori dichiarano che non esiste nessun conflitto di interessi.

\section{Bibliografia}

[1] Roulson J, Benbow EW, Hasleton PS. Discrepancies between clinical and autopsy diagnosis and the value of post mortem histology; a meta-analysis and review. Histopathology 2005; 47(6):551-9.

[2] Panella M, Kozel D, Marchisio S, Sarasino D, Pavanello PP, Jussich G, et al. Autopsy today: an obsolete practice or an instrument for improving the quality of health care? Pathologica 2000;92(2):58-64.

[3] Cabot RC. Diagnostic pitfalls identified during a study of three thousand autopsies. JAMA 1912;59:2295-8.

[4] Goldman L, Sayson R, Robbins S, Cohn LH, Bettmann M, Weisberg $M$. The value of the autopsy in three medical eras. N Engl J Med 1983;308(17):1000-5.

[5] Battle RM, Pathak D, Humble CG, Key CR, Vanatta PR, Hill RB, et al. Factors influencing discrepancies between premortem and postmortem diagnoses. JAMA 1987;258(3):339-44.

[6] Shojania KG, Burton EC, MCDonald KM, Goldman L. Changes in rates of autopsy-detected diagnostic errors over time: a systematic review. JAMA 2003;289(21):2849-56.

[7] Boers M, Nieuwenhuyzen Kruseman AC, Eulderink F, Hermans J, Thompson J. Value of autopsy in internal medicine: a 1-year prospective study of hospital deaths. Eur J Clin Invest 1988; 18(3):314-20.

[8] Ferguson RP, Burkhardt L, Hennawi G, Puthumana L. Consecutive autopsies on an internal medicine service. South Med J 2004;97(4):335-7.

[9] Bernicker EH, Atmar RL, Schaffner DL, Greenberg SB. Unanticipated diagnoses found at autopsy in an urban public teaching hospital. Am J Med Sci 1996;311(5):215-20.

[10] Durning S, Cation L. The educational value of autopsy in a residency training program. Arch Intern Med 2000;160(7): 997-9.

[11] Britton M. Diagnostic errors discovered at autopsy. Acta Med Scand 1974;196(3):203-10.

[12] Aalten CM, Samson MM, Jansen PA. Diagnostic errors; the need to have autopsies. Neth J Med 2006;64(6):186-90.

[13] Kotovicz F, Mauad T, Saldiva PH. Clinico-pathological discrepancies in a general university hospital in São Paulo. Brazil Clinics 2008;63(5):581-8.

[14] Gibson TN, Shirley SE, Escoffery CT, Reid M. Discrepancies between clinical and postmortem diagnoses in Jamaica: a study from the University Hospital of the West Indies. J Clin Pathol 2004;57(9):980-5.

[15] Maris C, Martin B, Creteur J, Remmelink M, Piagnerelli M, Salmon I, et al. Comparison of clinical and post-mortem findings in intensive care unit patients. Virchows Arch 2007;450(3): 329-33.

[16] Cameron HM, McGoogan E, Watson H. Necropsy: a yardstick for clinical diagnoses. Br Med J 1980;281(6246):985-8.

[17] Landefeld CS, Chren MM, Myers A, Geller R, Robbins S, Goldman L. Diagnostic yield of the autopsy in a university hospital and a community hospital. N Engl J Med 1988;318(19):1249-54. 